\title{
A new instrument of suction support adapter system for epicardial radiofrequency ablation
}

\author{
Shinichi Kadoya, MD, Go Watanabe, MD, Yoshinao Koshida, MD, Makoto Oda, MD, and \\ Shojiro Yamaguchi, MD
}

\begin{abstract}
Objective: Recently, epicardial ablation on a beating heart has been conducted as a minimally invasive surgical procedure for atrial fibrillation. We have developed a suction support adapter with the aim of ensuring contact between the ablation probe and the atrial wall to obtain transmural coagulation, and we evaluated the effectiveness of the adapter in electrophysiologic and histopathologic procedures.
\end{abstract}

\begin{abstract}
Methods: To ensure contact between the radiofrequency ablation probe and the atrial wall, we designed and fabricated a suction support adapter. Twelve pigs were used in this study. A comparison was made between a group in which the ablation probe alone was used (group $\mathrm{C}, \mathrm{n}=6$ ) and a group in which the ablation probe was used with the adapter (group $S, n=6$ ). In both groups, epicardial ablation was conducted on a beating heart. An electrophysiologic evaluation was conducted to determine whether there was electrical isolation. Histopathologic evaluations were performed to identify the range of coagulation in the resected atrial wall specimens.
\end{abstract}

Results: The coagulation range was significantly deeper in group $\mathrm{S}$ than in group $\mathrm{C}$, and the breadth was significantly narrower. Transmural coagulation and electrical isolation of the atrial wall were seen in 1 case in group $\mathrm{C}$ and in all cases in group $\mathrm{S}$.

Conclusions: Using the suction support adapter, we were able to maintain good contact between the ablation probe and the atrial wall. This device is thus thought to be useful for the safe and reliable performance of epicardial ablation on a beating heart. (J Thorac Cardiovasc Surg 2010;139:1618-22)

Cox and associates introduced the maze procedure for surgical treatment of atrial fibrillation in $1991 .^{1}$ Since that time, modifications have been added to create the maze III procedure, and various other modified procedures have been devised. $^{2-4}$ Ablation has been conducted using cryothermia, radiofrequency $(\mathrm{RF})$, or microwave energy as a technique to replace incision and suturing of the atrial wall in the maze procedure. With ablation using each of these different types of energy, transmural linear coagulation of the atrial wall can be performed rapidly, producing an electrical block.

In recent years, the epicardial approach has been used for ablation on the beating heart, and surgical treatment for atrial fibrillation has become less invasive. With an epicardial approach on the beating heart, however, it is difficult to ensure good contact between the ablation probe and the atrial wall, and the area of atrial wall ablation varies. In the present study, we developed a suction support adapter to reliably attach a unipolar RF ablation probe and conducted epicardial

From the Department of General and Cardiothoracic Surgery, Kanazawa University School of Medicine, Kanazawa, Japan.

Disclosures: None.

Received for publication July 28, 2009; revisions received Sept 18, 2009; accepted for publication Oct 8, 2009; available ahead of print Dec 28, 2009.

Address for reprints: Shinichi Kadoya, MD, Department of General and Cardiothoracic Surgery, Kanazawa University School of Medicine, 13-1 Takaramachi, Kanazawa, Ishikawa 920-0861, Japan (E-mail: s-kadoya@ja2.so-net.ne.jp). 0022-5223/\$0.00

Published by Elsevier Inc. on behalf of The American Association for Thoracic Surgery

doi:10.1016/j.jtcvs.2009.10.019 ablation on the atrial wall with beating pig hearts. The effects were then evaluated electrophysiologically and histopathologically.

\section{MATERIALS AND METHODS}

\section{Ablation Device and Suction Support Adapter}

The Cobra surgical probe (Estech Inc, San Ramon, Calif) was used as an RF ablation device. A suction support adapter was developed to ensure that the ablation probe remained in good contact with the atrial wall. The material used for the suction support adapter was a cylindrical silicone tube $8 \mathrm{~mm}$ in diameter with a thickness of $1 \mathrm{~mm}$. The ablation probe was fitted inside the silicone tube. Seven electrodes, $12 \mathrm{~mm}$ in width, were attached to the ablation probe, and an elliptical side hole was made in the silicone tube corresponding to the ablation points of the two leading electrodes, allowing ablation to be conducted (Figure 1). A suction tube was connected to the silicone tube, and the side hole could be suctioned against the atrial wall by applying continuous suction to the suction tube during ablation (Figure 2). Ablation was performed after confirming that the tube probe was in good contact with the atrial wall with suction pressure of -200 mbar.

\section{Experimental Animals}

Twelve pigs weighing 30 to $40 \mathrm{~kg}$ (mean, $33.5 \pm 3.2 \mathrm{~kg}$ ) were used. There were 2 experimental groups: group $C(n=6)$, in which Cobra device alone was used, and group $S(n=6)$, in which the suction support adapter was used with the Cobra device.

All animals received humane care in accordance with the "Principles of Laboratory Animal Care", formulated by the National Society for Medical Research and the "Guide for the Care and Use of Laboratory Animals," prepared by the Institute of Laboratory Animal Resources, National Research Council, and published by the National Academy Press, revised in 1996. 


\section{Abbreviations and Acronyms}

$\mathrm{RF}=$ radiofrequency

$\mathrm{TTC}=$ triphenyltetrazolium chloride
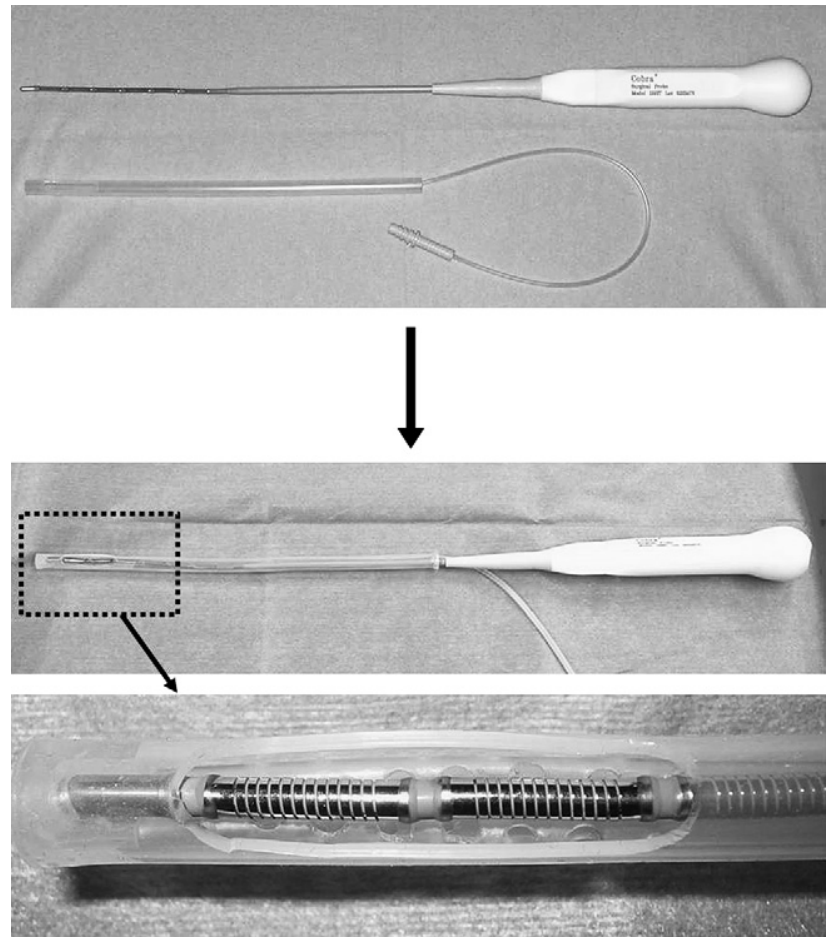

FIGURE 1. Cobra surgical probe and suction support adapter.

\section{Ablation Technique}

Pigs were given intramuscular injections of ketamine hydrochloride (20 $\mathrm{mg} / \mathrm{kg}$ body weight) and underwent endotracheal intubation. Anesthesia was maintained with inhalation administration of halothane $(0.5 \%-$ $1.5 \%)$, and intravenous administration of pancuroniumbromide $(0.1 \mathrm{mg} /$ $\mathrm{kg}$ ) was given as a muscle relaxant for inactivation. With the animal lying

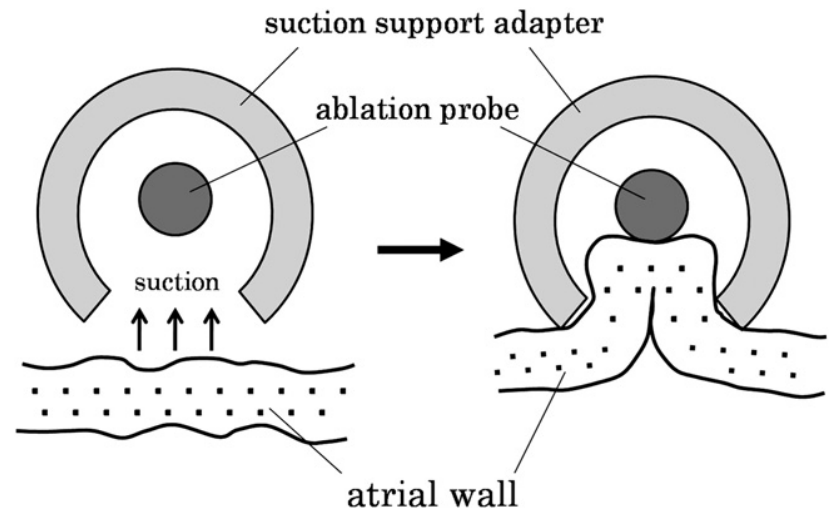

FIGURE 2. Schema of suction support adapter. With the use of the suction support adapter, the atrial wall folds into an inverted-U shape and is suctioned into the silicone tube. on its right side, the chest was opened with a lateral incision made at the left fifth intercostal space. An incision was then made in the pericardium and the left atrial wall was exposed so that ablation could be performed. Heparin (200 units/kg) was administered intravenously to prevent thrombus formation within the atrium. An ablation line of approximately $5 \mathrm{~cm}$ was set so that part of the left atrial auricle could be electrically isolated (Figure 3). In group $\mathrm{C}$, the ablation probe was placed directly on the ablation line. In group $\mathrm{S}$, the ablation probe was fitted with the suction support adapter and suctioned onto the ablation line with continuous suction of -200 mbar from the suction tube. In both groups, the ablation temperature was set at $80^{\circ} \mathrm{C}$ and the ablation time was set at 90 seconds. Ablation was conducted from the epicardium while the heart was beating.

\section{Electrophysiologic Findings}

After ablation, stimulating electrode S and bipolar electrode A1 were set inside the isolation area, and bipolar electrode A2 was set outside the isolation area. Pacing was conducted through stimulating electrode $\mathrm{S}$ set at 120 beats/min, $15 \mathrm{~mA}$, and an electrocardiogram was taken with electrodes $\mathrm{A} 1$ and $\mathrm{A} 2$ on the surface of the heart and a limb lead (Figure 3). Electrical isolation was successful when electrode A1 was synchronized with the pacing electrode, the electrocardiogram with electrode A2, and the limb lead was not synchronized.

\section{Gross Evaluation with 1\% Triphenyltetrazolium chloride (TTC) Staining of Cardiac Muscle}

After ablation was completed, animals were humanely killed with intravenous administration of pentobarbital and potassium chloride. The left atrial wall, including the ablation site, was then excised. Immediately after removal, an incision was made in the direction perpendicular to the ablation line in the portion where the atrial wall was thickest. Cross-sectional sections were stained with $1 \%$ TTC and the range of coagulation was evaluated. Atrial wall thickness and depth and breadth (millimeters) of the coagulation were measured in the coagulated portions.

\section{Histopathologic Evaluation of Cardiac Muscle}

Extirpated specimens contralateral to the TTC stain were fixed in $10 \%$ formalin and sections $(2 \mu \mathrm{m})$ were prepared in a direction perpendicular

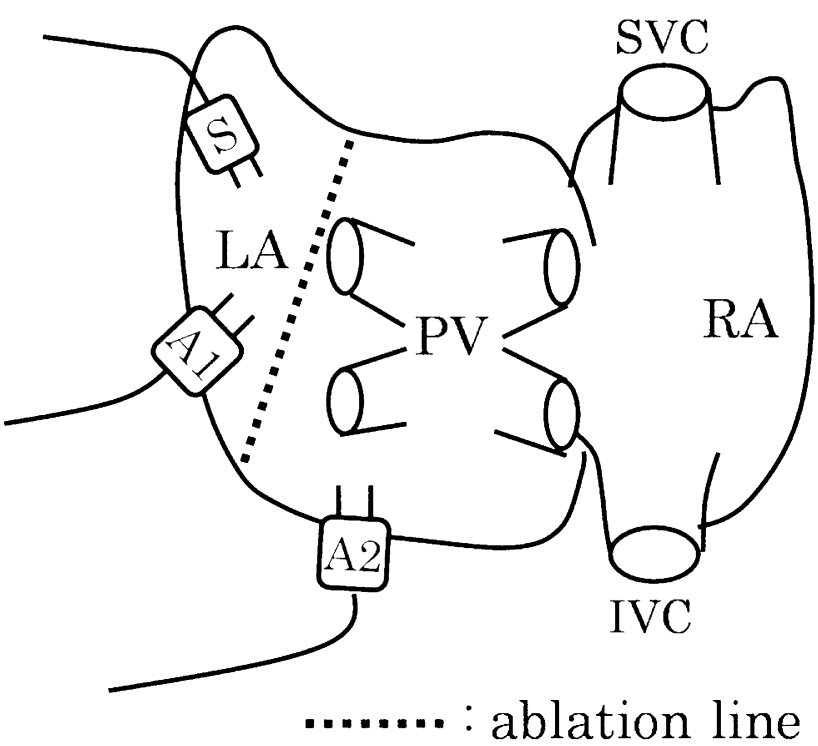

FIGURE 3. Schema of the epicardial ablation line (viewed from dorsal side). $S V C$, Superior vena cava; $I V C$, inferior vena cava; $R A$, right atrium; $L A$, left atrium; $P V$, pulmonary veins; $A 1, A 2$, and $S$, electrodes. 
to the ablation line. These sections were stained with azan and observed under a light microscope to assess the range of coagulation.

\section{Statistical Analysis}

SPSS (version 10.1.3) software (SPSS, Inc, Chicago, Ill) was used in statistical analysis. Median values and ranges were calculated for the depth and breadth of the coagulation range, and Wilcoxon sum rank test was used to test for differences between the groups. Analysis was conducted by $\chi^{2}$ test for the presence of transmural coagulation in the 2 groups.

\section{RESULTS}

\section{Intraoperative Assessment of the Effects of the} Suction Support Adapter

During the ablation procedure, the ablation probe could be steadily maintained against the atrial wall with the use of the suction support adapter, with almost no gap, despite the beating of the heart. When the suction support adapter was not used, however, some gaps occurred between the atrial wall of the beating heart and the ablation probe. No serious complications, such as damage to the tube from the heat load, popping of the atrial wall, or damage to the surrounding organs, occurred during the procedure in either group. No thrombosis within the atrium was seen in either group.

\section{Electrophysiologic Evaluation}

In models in which there was successful electrical isolation from ablation, electrode A1 in the isolated region was synchronized with the pacing electrode, and the electrocardiogram waveforms of electrode A2 and the limb lead outside the isolated region were not synchronized (Figure 4). In group $\mathrm{C}$, successful electrical isolation was seen in only 1 of 6 cases, the case with transmural coagulation; in the other 5 cases, electrical isolation was not successful. In group $\mathrm{S}$, there was successful electrical isolation in all cases.

A1

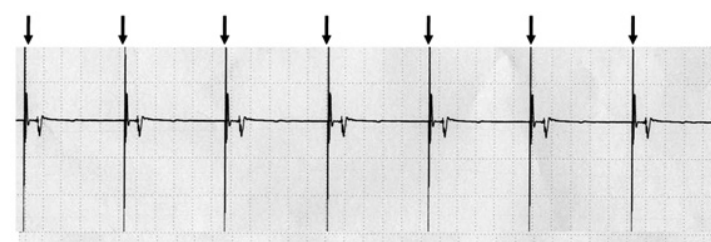

A2

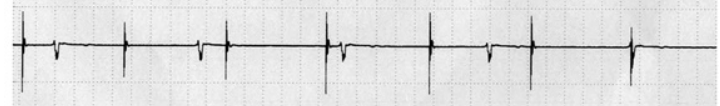

Limb lead

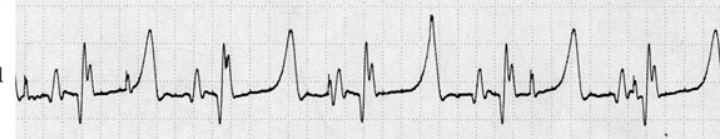

FIGURE 4. Electrophysiologic evaluation. In the group in which the suction support adapter was used, electrode A1 inside the isolation area was synchronized with the stimulus electrode (arrows) and electrode A2 and the limb lead outside the isolation area were not synchronized with the stimulus electrode (arrows). This indicates that electrical isolation occurred.

\section{Gross Myocardial Findings}

In both groups, the ablation line on the epicardial surface was discolored immediately after ablation, but the border with the healthy tissue was more distinct in group S. On the endocardial surface, distinct border discoloration was seen in all cases in group $\mathrm{S}$ and transmural coagulation was confirmed, but in group $\mathrm{C}$, no changes were seen on the endocardial surface.

Normal cardiac muscle was stained red with TTC, and a distinct border was seen with a region of yellowish coagulation (Figure 5, $A$ and $B$ ). The thickness of the atrial wall was not significantly different between the 2 groups. The coagulation range was significantly deeper in group $S$ than in group $\mathrm{C}$, and the breadth was significantly narrower. Transmural coagulation was seen in 1 case in group $\mathrm{C}$ and in all cases in group $\mathrm{S}$ (Table 1).

\section{Histopathologic Findings in Cardiac Muscle}

In the cardiac muscle specimens stained with azan, the region of coagulation was stained blue. Transmural coagulation was seen in only 1 of the 6 cases in group C. Transmural coagulation was seen in all 6 cases in group $\mathrm{S}$ (Figure 5, $C$ and $D$ ).

\section{DISCUSSION}

In recent years, advances have been made in ablation therapy for atrial fibrillation, and various methods have been devised for the approach, ablation site, and type of energy. In 1998, Haïssaguerre and associates ${ }^{5}$ indicated that repetitive atrial premature beat with a short coupling interval is a trigger for paroxysmal atrial fibrillation; they devised a method for electrically isolating the site of origin of the atrial premature beat within the pulmonary vein by ablating the opening of the pulmonary vein with a percutaneous catheter. Surgical procedures have also become more common to electrically isolate the origin of the atrial premature beat within the pulmonary vein by cryoablation or RF ablation in surgical treatments for atrial fibrillation. ${ }^{6,7}$

One issue in epicardial ablation on a beating heart is assuring that the ablation probe remains in contact with the atrial wall as the heart beats. Bipolar RF ablation or unipolar RF ablation using a fixed support instrument has been reported to reliably maintain the ablation probe against the beating atrial wall. ${ }^{8,9}$ Recently, coronary artery bypass grafts have been conducted with a beating heart, and the introduction of a suction type stabilizer made fixation to the ventricular wall possible. It has thus become safer and simpler to conduct coronary anastomosis while the heart is beating. ${ }^{10}$ If epicardial ablation can be conducted while the heart is beating, with the chosen site reliably immobilized during surgery for atrial fibrillation, establishment of a minimally invasive technique is expected that does not require the heart to be stopped. 


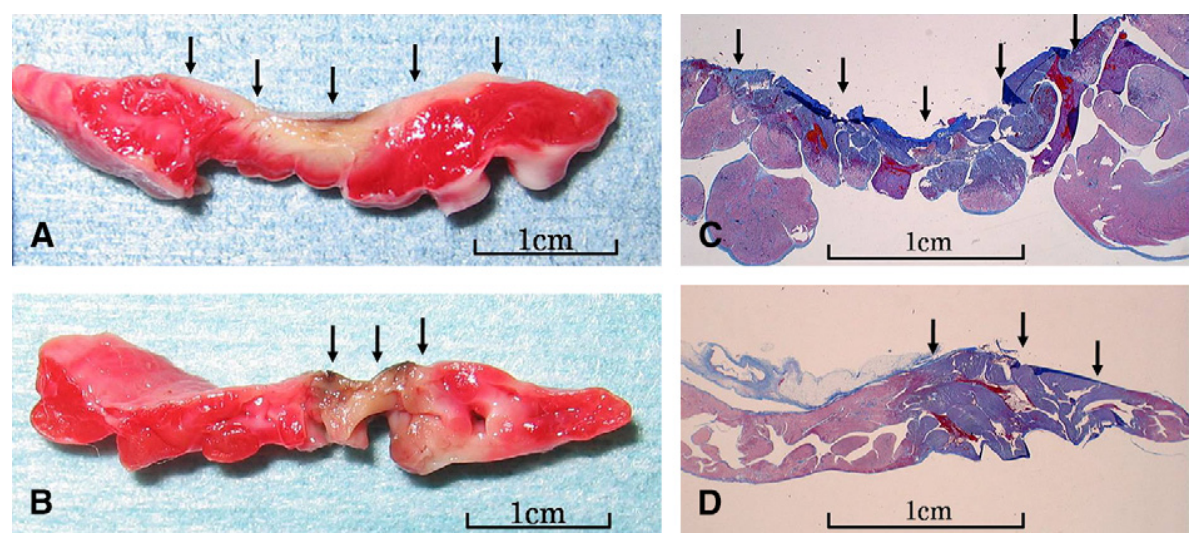

FIGURE 5. Normal cardiac muscle was stained red with triphenyltetrazolium chloride, and a distinct border was seen with a region of yellowish coagulation. A, In group C, the area of coagulation was large and transmurality was not seen (arrows). B, In group S, the area of coagulation was narrow and there was transmurality (arrows). In the azan-stained specimens, the area of coagulation was stained blue. C, In group C, the area of coagulation was large and transmurality was not seen (arrows). D, In group S, the area of coagulation was narrow and there was transmurality (arrows).

In the present study, we developed a suction support adapter for an RF ablation probe (Cobra surgical probe) with the aim of performing reliable transmural linear coagulation on a beating heart. This device has a silicone tube of 8 $\mathrm{mm}$ in diameter with a side hole for suction fitted over the ablation probe and connected to a suction tube. When ablation is performed with this device, the hole in the silicone tube is suctioned onto the atrial wall using by continuous suction, and it was demonstrated that the ablation probe and atrial wall remained in steady contact. It is difficult to use bipolar RF ablation devices on free wall portions of the atrium, but with our device, the ablation probe can be kept in steady contact with the beating atrium regardless of location, even during ablation conducted against the atrial free wall. Another major advantage of this device is that, unlike clamp-type devices, such as the Atricure device (Atricure Inc, Cincinnati, Ohio), ${ }^{8}$ linear ablation can be performed on a thin ablation line on a free wall. Thus it is easy to perform a technique in which a line can be made in addition to pulmonary vein isolation, with almost no effect on structures (coronary artery, ventricle) and other counter portions.

Progress has been made in endoscopic surgery techniques and devices, and clinical studies of epicardial ablation using a thoracoscope are occasionally conducted. ${ }^{11-13}$ By reducing damage to the thoracic wall, thoracoscopic surgery has

TABLE 1. Evaluations of the resected atrial wall specimens with $1 \%$ TTC stain

\begin{tabular}{lccc}
\hline & $\begin{array}{c}\text { Group C } \\
(\mathbf{n}=\mathbf{6})\end{array}$ & $\begin{array}{c}\text { Group S } \\
(\mathbf{n}=\mathbf{6})\end{array}$ & $\begin{array}{c}\boldsymbol{P} \\
\text { value }\end{array}$ \\
\hline Thickness of atrial wall $(\mathrm{mm})$ & $6.0(5.0-7.5)$ & $5.5(5.0-6.5)$ & .310 \\
Depth of the coagulation $(\mathrm{mm})$ & $3.0(2.5-5.5)$ & $5.5(5.0-6.5)$ & .015 \\
Breadth of the coagulation (mm) & $11.0(10.0-12.5)$ & $8.0(8.0-10.0)$ & .011 \\
Transmurality (no.) & 1 & 6 & .003 \\
\hline TTC, Triphenyltetrazolium chloride. & & &
\end{tabular}

attracted attention as a minimally invasive surgery with few postoperative respiratory complications and quick recovery. However, the visual field is limited in thoracoscopic surgery, as is the mobility of forceps, so that there is a need to develop an ablation device that is suited to thoracoscopic surgery. The developed suction support adapter is a silicone cylinder with a diameter of $8 \mathrm{~mm}$. The device is also flexible and can readily be applied to thoracoscopic surgery. Moreover, the ablation probe is completely covered by the silicone tube, except where it contacts the atrial wall, so that there is no risk of heat damage to surrounding tissue. Ablation can be safely performed, even with the limited visual field encountered during thoracoscopy. Moreover, the device can be attached to identically shaped probes that use other types of energy; it is therefore promising in the field of robotic surgery, another focus of recent attention. ${ }^{14}$

One further issue in epicardial ablation on a beating heart is the cooling effect from atrial blood flow. Using the suction support adapter developed in the present study, the atrial wall is folded into an inverted- $U$ shape and suctioned into the silicone tube. It is thought that this makes it more difficult for the cooling effect to reach the endocardium. The present results indicate that with the use of the suction support adapter, the area of coagulation was significantly narrower and went deeper. Transmurality was seen in 1 of 6 cases in group C, but in all cases in group S. This suction support adapter has the two advantages of ensuring contact with the atrial wall while the heart is beating and avoiding the cooling effects from atrial blood flow. It is thus considered to be an effective device for conducting sure transmural linear ablation.

The temperature and time settings for unipolar RF ablation in previous reports were $60^{\circ}$ to $80^{\circ} \mathrm{C}$ and 60 to $120 \mathrm{sec}$ onds for endocardial ablation. ${ }^{7,15}$ However, because there is no consensus on the proper value of these parameters, the settings for epicardial ablation vary. ${ }^{16,17}$ In general, it is thought that deeper coagulation can be achieved with longer 
ablation times, but excessive prolonging of ablation time carries the risk of producing serious complications, such as atrial thrombosis or atrial wall perforation. With reference to previous reports, ${ }^{15-17}$ we set temperature at $80^{\circ} \mathrm{C}$ and time at 90 seconds in the present study, and no complications with atrial thrombosis or atrial wall perforation were noted. Moreover, transmural coagulation was possible in all cases using the suction support adapter, and these are therefore considered to be good settings for unipolar RF ablation of the atrial wall in pigs. Optimal temperature and time settings for epicardial ablation of the atrial wall of humans with the heart beating have not yet been determined. Finding the best settings to achieve sure transmural coagulation without complications such as thrombosis or perforation is an issue for future studies. In the present study, we investigated the effects of ablation in the acute phase, but future studies will investigate the effects of ablation in the chronic phase.

\section{References}

1. Cox JL, Schuessler RB, Boineau JP. The surgical treatment of atrial fibrillation. I. Summary of the current concepts of mechanisms of atrial flutter and atrial fibrillation. J Thorac Cardiovasc Surg. 1991;110:402-5.

2. Cox JL, Boineau JP, Schuessler RB, Jaquiss RDB, Lappas DG. Modification of the maze procedure for atrial flutter and atrial fibrillation. I. Rationale and surgical results. J Thorac Cardiovasc Surg. 1995;110:473-84.

3. Kim KB, Huh JH, Kang CH, Ahn H, Sohn DW. Modifications of the Cox-Maze III procedure. Ann Thorac Surg. 2001;71:816-22.

4. Kosakai Y, Kawaguchi AT, Isobe F, Sasako Y, Nakano K, Eishi K, et al. Y. Cox maze procedure for chronic atrial fibrillation associated with mitral valve disease. J Thorac Cardiovasc Surg. 1994;108:1049-54.
5. Haïssaguerre M, Jaïs P, Shah DC, Takahashi A, Hocini M, Quiniou G, et al. Spontaneous initiation of atrial fibrillation by ectopic beats originating in the pulmonary veins. N Engl J Med. 1998;339:659-66.

6. Van Belle Y, Janse P, Rivero-Ayerza MJ, Thornton AS, Jessurun ER, Theuns D, et al. Pulmonary vein isolation using an occluding cryoballoon for circumferential ablation: feasibility, complications, and short-term outcome. Eur Heart J. 2007; 28:2231-7.

7. Benussi S, Nascimbene S, Agricola E, Calori G, Calvi S, Caldarola A, et al. Surgical ablation of atrial fibrillation using the epicardial radiofrequency approach: mid-term results and risk analysis. Ann Thorac Surg. 2002;74: 1050-7.

8. Prasad SM, Maniar HS, Schuessler RB, Damiano RJ Jr. Chronic transmural atrial ablation by using bipolar radiofrequency energy on the beating heart. $J$ Thorac Cardiovasc Surg. 2002;124:708-13.

9. Ishikawa S, Oki S, Muraoka M, Kashiwabara K, Morishita Y. Support adapter for radiofrequency ablation probe: experimental study. ANZ J Surg. 2005;75:157-9.

10. Pym J. Off-pump arterial grafting: 125 cases using the Medtronic-Utrecht Octopus. Eur J Cardiothorac Surg. 1999;16:S88-94.

11. Koistinen J, Valtonen M, Savola J, Airaksinen J. Thoracoscopic microwave ablation of atrial fibrillation. Interact Cardiovasc Thorac Surg. 2007;6:695-8.

12. Grandmougin D, Tiffet O. Video-assisted thoracoscopic epicardial ablation of left pulmonary veins for lone permanent atrial fibrillation. Interact Cardiovasc Thorac Surg. 2007;6:136-8.

13. Sirak J, Jones D, Sun B, Sai-Sudhakar C, Crestanello J, Firstenberg M. Toward a definitive, totally thoracoscopic procedure for atrial fibrillation. Ann Thorac Surg. 2008;86:1960-4.

14. Ota T, Degani A, Schwartzman D, Zubiate B, McGarvey J, Choset H, et al. A novel highly articulated robotic surgical system for epicardial ablation. Conf Proc IEEE Eng Med Biol Soc. 2008;1:250-3.

15. Melo J, Adragão P, Neves J, Ferreira M, Timóteo A, Santiago T, et al. Endocardial and epicardial radiofrequency ablation in the treatment of atrial fibrillation with a new intra-operative device. Eur J Cardiothorac Surg. 2000;18:182-6.

16. Ishikawa S, Oki S, Muraoka M, Oshima K, Kashiwabara K, Morishita Y. Epicardial radiofrequency ablation on a beating heart: an experimental study. Ann Thorac Cardiovasc Surg. 2005;11:21-4.

17. Inoue Y, Kiso I, Takahashi R, Mori A, Motogami K. Beating-heart epicardial radiofrequency ablation: optimal temperature setting. Ann Thorac Surg. 2004;78: 308-12. 